\title{
Myocardin/microRNA-30a/Beclin1 signaling controls the phenotypic modulation of vascular smooth muscle cells by regulating autophagy
}

Danyang Shi ${ }^{1,2,4}$, Jinhua Ding ${ }^{1,2,4}$, Shouqiang Xie ${ }^{1,2,4}$, Lei Huang ${ }^{1,2}$, Hongmin Zhang ${ }^{1,2}$, Xiaojie Chen ${ }^{1,2}$, Xuejun Ren ${ }^{3}$, Sa Zhou ${ }^{1,2}$,

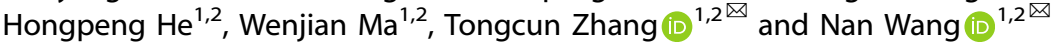

(c) The Author(s) 2022, corrected publication 2022

Upon vascular injury, vascular smooth muscle cells (VSMCs) change from a contractile phenotype to a synthetic phenotype, thereby leading to atherogenesis and arterial restenosis. Myocardin (MYOCD) is essential for maintaining the contractile phenotype of VSMCs. Deletion of MYOCD in VSMCs triggers autophagy. However, the molecular mechanism underlying the effect of MYOCD on autophagy is not clear. In this study, knockdown of MYOCD in human aortic VSMCs (HA-VSMCs) triggered autophagy and diminished the expression of SMC contractile proteins. Inhibition of autophagy in MYOCD-knockdown cells restored the expression of contractile proteins. MYOCD activated the transcription of miR-30a by binding to the CArG box present in its promoter, as confirmed by luciferase reporter and chromatin immune coprecipitation assays, while miR-30a decreased the expression of autophagy protein-6 (ATG6, also known as beclin1) by targeting its 3'UTR. Restoring the expression of miR-30a in MYOCDknockdown cells upregulated the levels of contractile proteins. Treatment of VSMCs with platelet-derived growth factor type BB (PDGF-BB) resulted in the transformation of VSMCs to a proliferative phenotype. A low level of miR-30a was observed in PDGF-BBtreated HA-VSMCs, and re-expression of miR-30a led to a decrease in proliferative marker expression. Furthermore, using a wire injury mouse model, we found that miR-30a expression was significantly downregulated in the arterial tissues of mice and that restoration of miR-30a expression at the injured site abolished neointimal formation. Herein, MYOCD could inhibit autophagy by activating the transcription of miR-30a and that miR-30a-mediated autophagy defects could inhibit intimal hyperplasia in a carotid arterial injury model.

Cell Death and Disease (2022)13:121; https://doi.org/10.1038/s41419-022-04588-0

\section{INTRODUCTION}

The switching of vascular smooth muscle cells (VSMCs) from a contractile, quiescent or differentiation phenotype to an adverse, synthetic, proliferative or dedifferentiation phenotype plays an important role in the pathogenesis of various vascular proliferative diseases, including atherosclerosis, restenosis, and aneurysm. Inappropriate VSMC activation and proliferation are critical causes of neointima formation, stenosis and obstructions of vessels. The proliferative phenotype of VSMCs is characterized by proliferation and migration, as well as decreased levels of contractile marker proteins such as alpha smooth muscle actin (ACTA2, alpha-SMA), smooth muscle 22 alpha (SM22a) and smooth muscle myosin heavy chain (SMMHC). Understanding the potential mechanisms of VSMC phenotypic switching provides a new therapeutic strategy for the treatment of vascular proliferative diseases.

Myocardin (MYOCD) is expressed specifically in cardiac myocytes and VSMCs and plays a critical role in cardiovascular development [1]. It is a serum response factor (SRF)-dependent cofactor and can activate the expression of smooth muscle- specific genes by binding to SRFs on CArG box-containing target genes [2]. During postnatal development, MYOCD is essential for maintaining homeostasis in the vascular system and visceral tissues. MYOCD expression represents a contractile and differentiated SMC phenotype. Deletion of MYOCD, however, represents a synthetic and dedifferentiated phenotype, a hallmark of atherosclerosis [3]. Using tamoxifen-treated SMMHC-Cre ${ }^{E R T 2}$ / Myocd $^{F / F}$ conditional mutant mice, Jianhe Huang et al. confirmed that loss of MYOCD markedly decreased the expression of SMC contractile proteins and induced cell autonomous autophagy [4]. This phenomenon suggests a new feature of MYOCD in regulating autophagy. However, it is not clear why the loss of MYOCD causes autophagy activation in SMCs.

Increasing evidence has confirmed the critical role of autophagy in the smooth muscle cell phenotype switch. Autophagy is one of the key degradation systems devoted to degrading and recycling misfolded proteins and damaged or aged organelles. Autophagy activation can be observed in VSMCs in vascular diseases, including atherosclerosis [5], hypertension [6] and dissecting

\footnotetext{
${ }^{1}$ College of Biotechnology, Tianjin University of Science and Technology, Tianjin, China. ${ }^{2}$ Key Laboratory of Industrial Fermentation Microbiology, Ministry of Education and Tianjin, Tianjin, China. ${ }^{3}$ Department of Cardiology, Beijing Anzhen Hospital Affiliated to Capital Medical University, Beijing, China. ${ }^{4}$ These authors contributed equally: Danyang Shi, Jinhua Ding, Shouqiang Xie. ${ }^{凶}$ email: tony@tust.edu.cn; wn929@tust.edu.cn Edited by Professor Gian Maria Fimia.
}

Received: 7 June 2021 Revised: 4 January 2022 Accepted: 27 January 2022 Published online: 08 February 2022 
aortic aneurysms (AAs) [7]. Various stimuli and stressors, such as reactive oxygen and lipid species [8], proinflammatory cytokines $[9,10]$ and shear stress [11], have been shown to induce autophagy in VSMCs. It has been reported that various stimuli, including secreted protein sonic hedgehog [12], tumor necrosis factor-a (TNF-a) [9], platelet-derived growth factor type BB (PDGFBB) [10], and nicotine [13], inhibit the expression of key VSMC contractile genes and induce dedifferentiation of VSMCs, as well as the activation of autophagy. Activation of autophagy induced by PDGF-BB results in the degradation of contractile proteins, leading to the dedifferentiation phenotype of VSMCs [10]. However, it is still unclear how activation of autophagy regulates the expression of synthetic proteins in VSMCs.

MicroRNAs (miRNAs) are a class of endogenous, 22-24 nucleotide noncoding RNAs that function as posttranscriptional regulators of gene expression. MiRNAs have been found to participate in a variety of biological processes ranging from critical physiological functions to the pathophysiology of many diseases. Recent studies have confirmed the important role of miRNAs in the posttranslational orchestration of autophagy-related genes. MiRNA-30a has been reported to inhibit autophagy by directly targeting beclin 1 in various types of cells [14-16]. However, the role of miRNA-30a in the phenotypic switch of vascular smooth muscle cells is still unknown.

In this study, we revealed a new mechanism by which MYOCD regulated the expression of smooth muscle contractile genes. We found that MYOCD could maintain the expression of SMC contractile proteins by upregulating miRNA-30a-5p and inhibiting VSMC autophagy. We have also demonstrated for the first time that miRNA-30a-5p plays an important role in regulating the phenotypic switch of VSMCs.

\section{RESULTS \\ Knockdown of MYOCD triggered autophagy in HA-VSMCs}

To test whether MYOCD can regulate the autophagy response, MYOCD was knocked down in HA-VSMCs by transfection with an shRNA MYOCD plasmid. Knockdown of MYOCD was confirmed by western blotting (Fig. 1A), and a shRNA\#3 plasmid against MYOCD was used in subsequent experiments. The expression of contractile proteins ACTA2 and SM22a was decreased in HA-VSMCs transfected with shRNA specifically targeting MYOCD compared with control shRNA (Fig. 1B), whereas the levels of proliferational markers Osteopontin (OPN) and proliferating cell nuclear antigen (PCNA) were increased (Fig. 1C), which was consistent with previous reports [3]. Knockdown of MYOCD significantly increased the number of EdU-positive cells (Fig. 1D). Knockdown of MYOCD decreased the expression of p62 and increased the expression of beclin 1 and the conversion of LC3-I to LC3-II (Fig. 1E). Cotreatment of the lysosomal inhibitor bafilomycin A1 and shMYOCD led to higher accumulation of LC3-II and p62 compared to shMYOCD group (Fig. 1F), suggesting that knockdown of MYOCD increased the autophagic flux. Moreover, the formation of autophagosomes was observed in HA-VSMCs by transfecting cells with a green fluorescent protein (GFP)-LC3 expression construct and quantifying the number of GFP-LC3 puncta per cell by fluorescence microscopy. More GFP-LC3 dots were found in HA-VSMCs transfected with shRNA against MYOCD compared to HA-VSMCs transfected with control shRNA, and treatment of bafilomycin A1 in MYOCD-knockdown cells further increased the number of GFPLC3 dots (Fig. 1G).

\section{Inhibition of autophagy in MYOCD-knockdown cells restored the expression of contractile proteins}

Next, we wanted to determine whether the expression of contractile proteins could be restored by 3-methyladenine (3$M A$, an autophagy inhibitor). HA-VSMCs were transfected with shMYOCD plasmid for $4 \mathrm{~h}$ and then treated with 3-MA for another
$48 \mathrm{~h}$. As shown in Fig. 2, knockdown of MYOCD upregulated the expression of beclin 1 and the ratio of microtubule-associated protein 1 light chain 3 alpha (LC3II/LC3I) and downregulated the level of p62, suggesting the activation of autophagy. The expression of the contractile proteins ACTA2 and SM22a was decreased in MYOCD knockdown cells. Treatment with 3-MA inhibited the autophagy induced by knockdown of MYOCD and restored the level of contractile proteins. These results showed that MYOCD could affect the expression of contractile proteins by regulating autophagy.

\section{MYOCD activated the transcription of miR-30a by binding to the CArG box}

MiRNA-30a-5p has been known to mediate beclin1 suppression by targeting the $3^{\prime}$ UTR of beclin1 [16]. Here, we presumed that MYOCD could positively regulate the expression of miR-30a-5p. To demonstrate this hypothesis, the level of miR-30a-5p was examined in MYOCD overexpression or knockdown cells by RT$P C R$. As shown in Fig. $3 A$ and $B$, overexpression of MYOCD led to an increase in miR-30a-5p, and knockdown of MYOCD led to a decrease in miR-30a-5p.

Bioinformatics analysis revealed that the promoter regions of miR-30a-5p contain a CArG box motif (CCTTAATGGG) (Fig. 3C). To confirm whether MYOCD can activate the transcription of miR$30 a-5 p$, a luciferase reporter vector containing the promoter regions of miR-30a-5p was constructed, and then the luciferase assay was performed in MYOCD overexpression or knockdown cells. As shown in Fig. 3D, MYOCD activated the transcription of the miR-30a-5p promoter, while mutation of the CArG box in the miR-30a-5p promoter abolished this activation. The activity of the miR-30a-5p promoter was weakened by MYOCD knockdown or PDGF-BB treatment (Fig. 3E and F). To further confirm the specific binding of MYOCD to the miR-30a-5p promoter, ChIP assays were performed in cells transfected with the MYOCD-myc vector using antibodies against myc. As shown in Fig. 3G, MYOCD enrichment at the CArG box region of the miR-30a-5p promoter was significantly enhanced. These data demonstrated that MYOCD could promote the transactivation of miR-30a-5p by binding to the CArG box motif of the miR-30a-5p promoter.

Since knockdown of MYOCD can inhibit the level of miR-30a-5p, we wanted to know whether recovery of miR-30a-5p expression in MYOCD-knockdown cells could restore the expression of contractile proteins. As shown in Fig. $3 \mathrm{H}$, miR-30a-5p mimics enhanced the expression of contractile proteins ACTA2 and SM22a and inhibited autophagy in MYOCD-knockdown cells.

\section{MiR-30a-5p decreased the expression of beclin 1 by targeting the 3'UTR of beclin 1}

To confirm that beclin1 is a target of miR-30a-5p in HA-VSMCs, cells were transfected with miR-30a-5p mimics, and then the expression of beclin1 was detected by western blotting. The results showed that miR-30a-5p significantly reduced the expression of beclin1 in HA-VSMCs (Fig. 4A). To confirm that miR-30a-5p can target the $3^{\prime}$-untranslated region (3'-UTR) of beclin 1 mRNA to suppress protein translation, the $3^{\prime}$-UTR of beclin1 containing miR$30 a-5 p$ binding sites (WT-BECN1-3'UTR) or mutation (MUT-BECN1$3^{\prime}$ UTR) was cloned into the pmirGLO vector (Fig. 4B), and this vector was cotransfected with the miR-30a-5p mimics in cells for $24 \mathrm{~h}$. As indicated in Fig. 4C, the overexpression of a miR-30a-5p mimic repressed the activity of luciferase fused with the WT- $3^{\prime}-$ UTR of beclin1 but had no effect on luciferase activity when the miR-30a-5p binding sites in the $3^{\prime}-$ UTR of beclin 1 were mutated. Consistent with other reports [14-16], our results also confirmed that miR-30a-5p could negatively regulate the level of beclin1 in HA-VSMCs by targeting the $3^{\prime}$-UTR of beclin1.

Then, we hypothesized that miR-30a-5p knockdown could activate autophagy and cause a decrease in the level of contractile proteins. As shown in Fig. 4D and E, the autophagic 
A

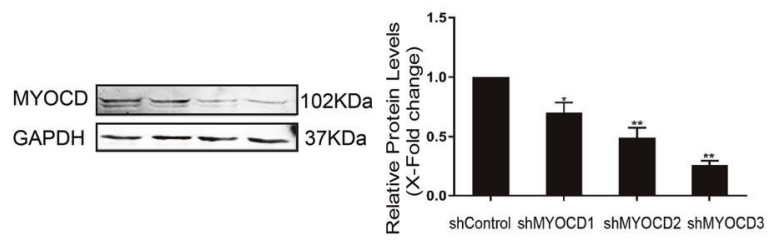

C
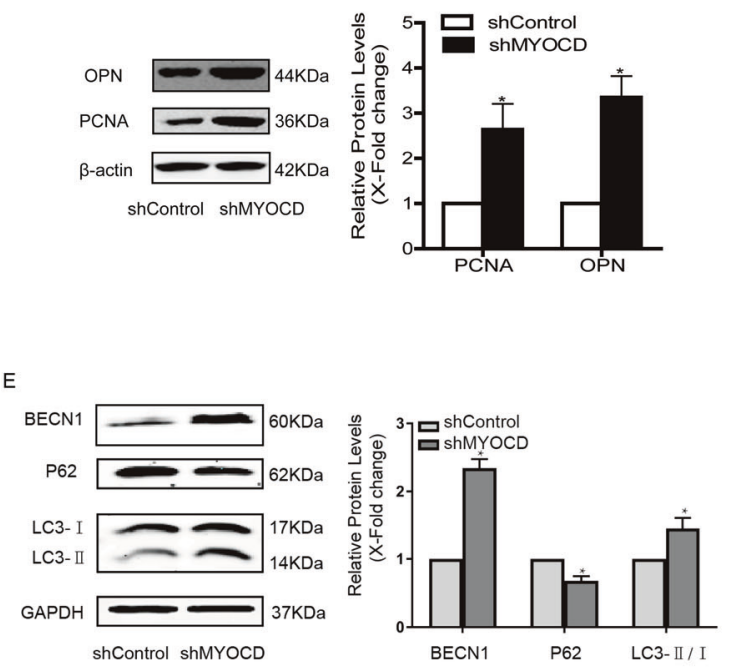

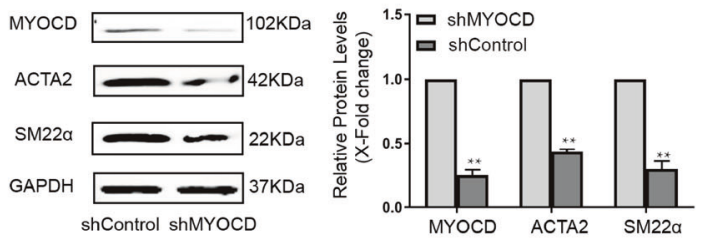

D
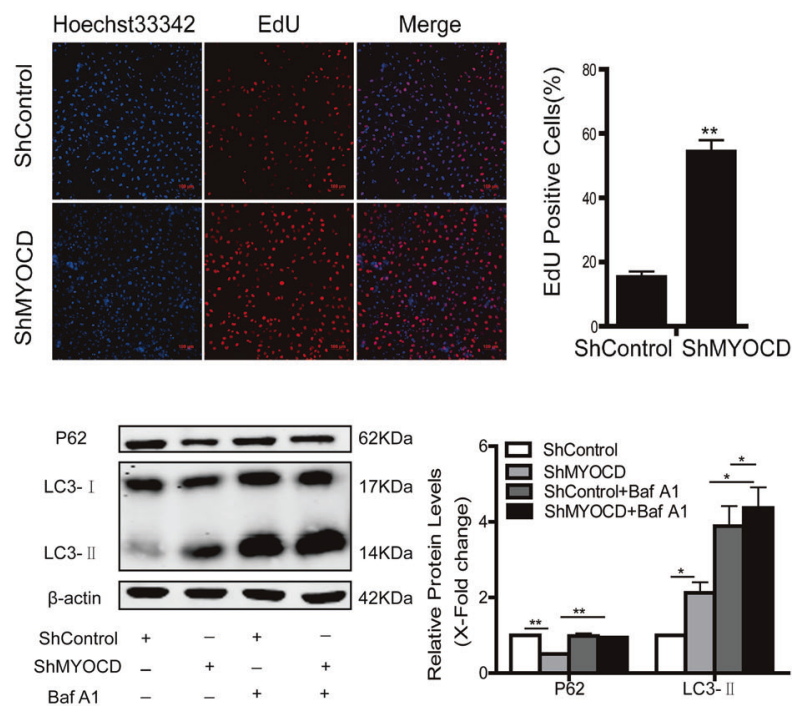
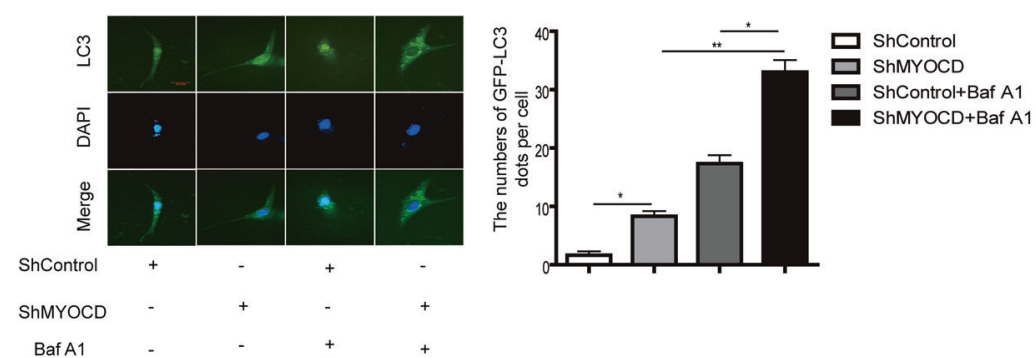

Fig. 1 Knockdown of MYOCD triggered autophagy in HA-VSMCs. A The expression of MYOCD was detected in HA-VSMCs transfected with shRNA specifically targeting MYOCD by western blotting $\left({ }^{*} P<0.05,{ }^{* *} P<0.01, n=3\right)$. B The expression of contractile proteins ACTA2 and SM22 was detected in MYOCD-knockdown HA-VSMCs by western blotting $\left.{ }^{* *} P<0.01, n=3\right)$. C The expression of OPN and PCNA was tested in MYOCD-knockdown HA-VSMCs by western blotting ( $\left.{ }^{*} P<0.05, n=3\right)$. D Cellular proliferation was evaluated in MYOCD-knockdown HA-VSMCs by EdU staining $(* * P<0.01, n=3)$. E The expression of beclin 1, P62, and LC3 was tested in MYOCD-knockdown HA-VSMCs by western blotting $\left({ }^{*} P<0.05, n=3\right)$. F Bafilomycin A1 $(0.1 \mu \mathrm{g} / \mathrm{mL})$ was added to control or MYOCD-knockdown HA-VSMCs $1 \mathrm{~h}$ prior to harvest, and accumulation of P62 and LC3 was measured by western blotting $\left({ }^{*} P<0.05, * * P<0.01, n=3\right)$. G Representative images and quantification of GFP-LC3 puncta in MYOCD-knockdown HA-VSMCs transfected with GFP-LC3 vector in the presence or absence of bafilomycin A1 $\left({ }^{*} P<0.05,{ }^{* *} P<0.01, n=3\right)$
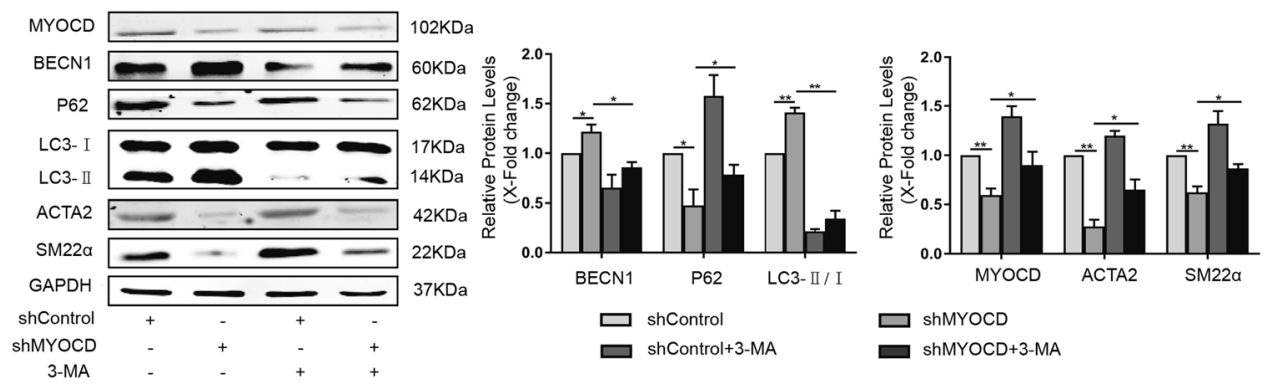

Fig. 2 Inhibition of autophagy in MYOCD-knockdown cells restored the expression of contractile proteins. HA-VSMCs were transfected with shMYOCD plasmid for $4 \mathrm{~h}$ and then treated with $5 \mathrm{mM} 3-\mathrm{MA}$ for another $48 \mathrm{~h}$. Western blot images and quantification in each group were shown $(* P<0.05, * * P<0.01, n=3)$. 
A

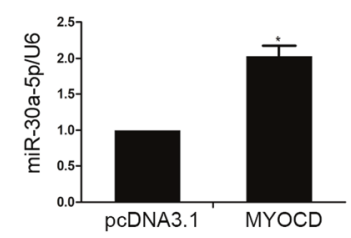

D

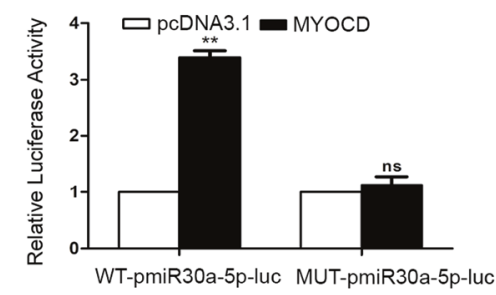

B

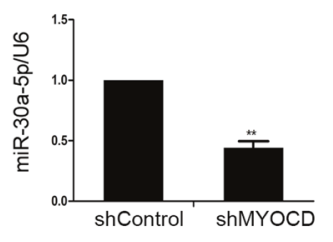

E
C

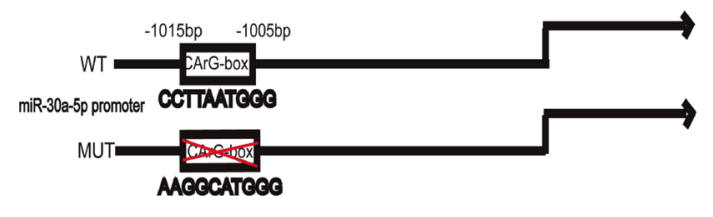

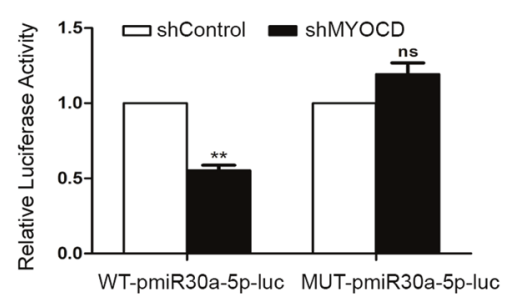

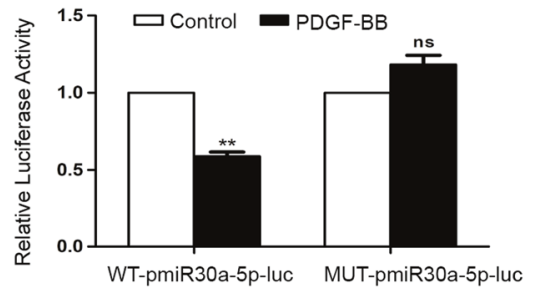

G

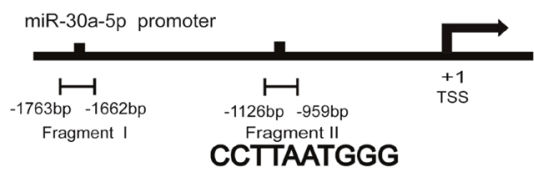

H
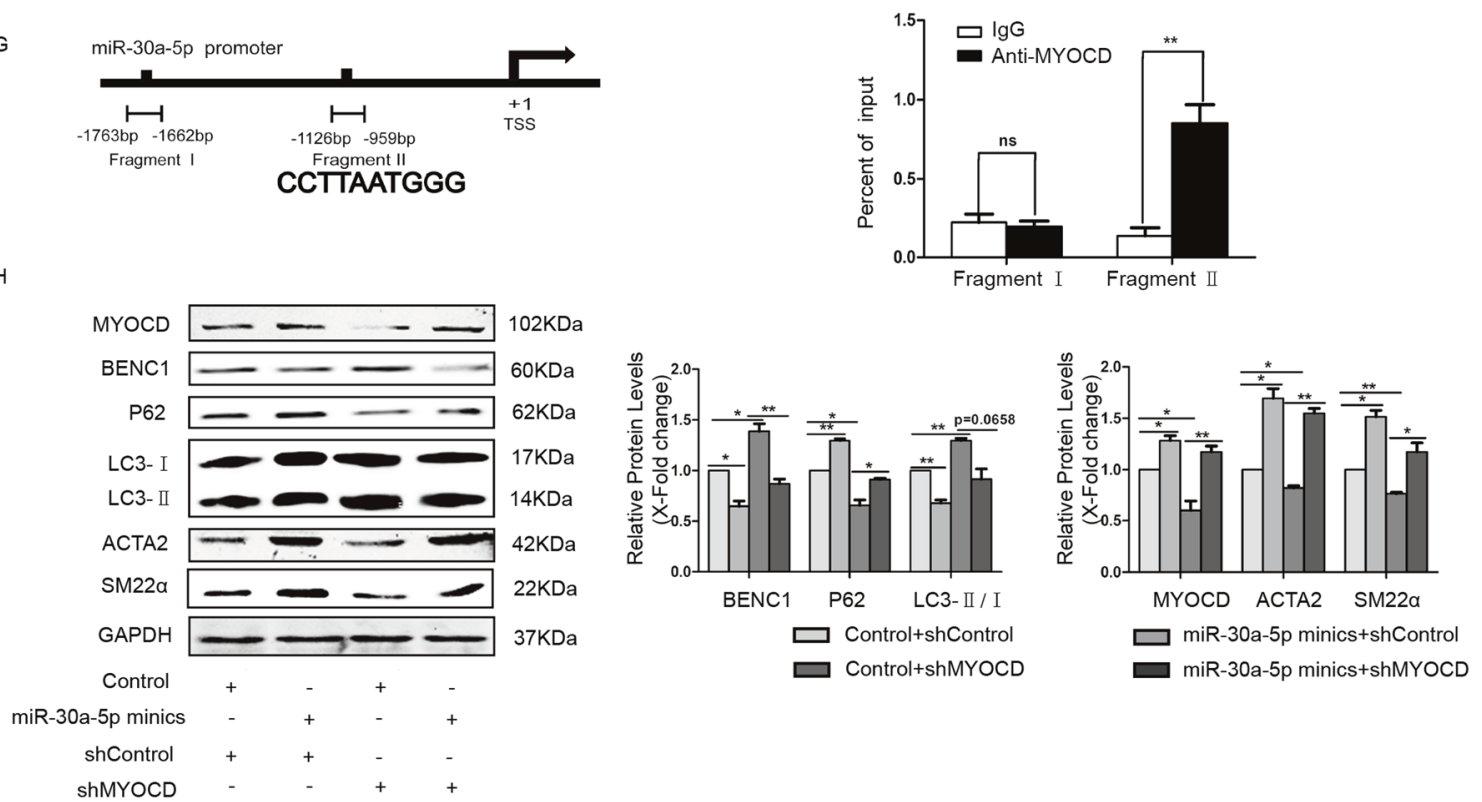

Fig. 3 MYOCD activated the transcription of miR-30a by binding to the CArG box. The level of miR-30a-5p was examined in MYOCD overexpression (A) or knockdown (B) cells by RT-PCR $\left(* P<0.05,{ }^{* *} P<0.01, n=3\right)$. C A schematic diagram of the miR-30a-5p promoter region represents wild-type and mutant CArG boxes. D Luciferase reporter plasmids together with pCDNA3.1-MYOCD plasmids or vectors were cotransfected into HA-VSMCs, and $24 \mathrm{~h}$ after transfection, luciferase activity was assayed $(* * P<0.01, n=3$ ). E HA-VSMCs were transfected with luciferase reporter plasmids together with shControl or shMYOCD plasmids for $24 \mathrm{~h}$, and then luciferase activity was assayed $(* * P<0.01, n=3$ ). F HA-VSMCs were transfected with luciferase reporter plasmids in the presence or absence of PDGF-BB for $24 \mathrm{~h}$, and then luciferase activity was assayed ( $\left.{ }^{*} P<0.01, n=3\right)$. G $48 \mathrm{~h}$ after transfection of pcDNA3.1-MYOCD-myc vector expression plasmids or vector plasmids, ChIP assays were performed using antibodies against myc (**P<0.01, $n=3)$. H MiR-30a-5p mimics were transfected into MYOCD knockdown cells, and then western blotting was performed $\left(* P<0.05,{ }^{*} P<0.01, n=3\right)$.

flux was measured in miR-30a-5p inhibitor-treated cells with/ without bafilomycin A1 by detecting the levels of p62 and LC3. The results showed that cotreatment of bafilomycin $A 1$ and miR-30a-5p inhibitor led to higher accumulation of LC3-II and p62 compared to miR-30a-5p inhibitor group, suggesting that miR-30a-5p inhibitor activates autophagy. Furthermore, decreased expression of the contractile proteins ACTA2 and SM22a was observed in cells transfected with the miR-30a-5p inhibitor (Fig. 4F and G). Incubation with 3-MA inactivated autophagy and enhanced the expression of contractile proteins in cells transfected with the miR-30a-5p inhibitor (Fig. 4F and G). MiR-30a-5p inhibitor also increased the expression of PCNA and OPN, while 3-MA treatment inhibited the expression of PCNA and OPN (Fig. 4H and I). More EDU positive cells were observed in miR-30a-5p inhibitor group, compared with Control group, while 3-MA treatment reversed this change caused by miR-30a-5p inhibitor (Fig. 4J and K).

\section{Inhibition of autophagy could maintain the contractile phenotype in PDGF-BB-stimulated HA-VSMCs}

PDGF-BB plays a major role in inducing the switch of VSMCs from the contractile phenotype to the proliferative phenotype. Here, PDGF-BB stimulation upregulated the expression of the proliferative markers OPN and PCNA, and downregulated the expression of ACTA2 and SM22a in HA-VSMCs (Fig. 5 A-C). The PDGF-BB-stimulated switch of HA-VSMCs into a proliferative phenotype was also confirmed by EdU staining (Fig. 5D and E). In line with previous studies [10], we also found that PDGF-BB triggered autophagy in HA-VSMCs, as confirmed by the increase in beclin1 protein and LC3II/LC3I and the decrease in P62 protein (Fig. 5F and G). PDGF-BB induced a significant increase in 

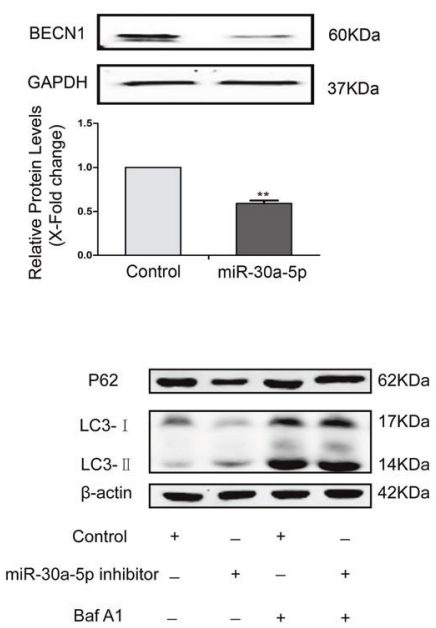

G

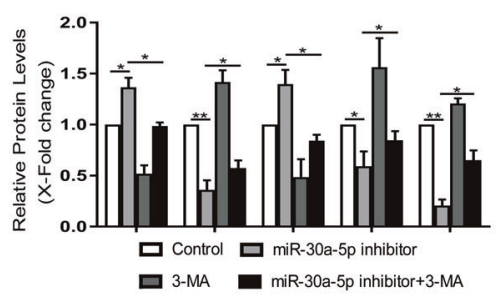

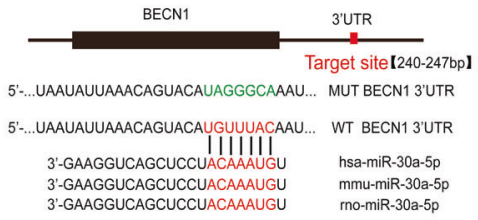
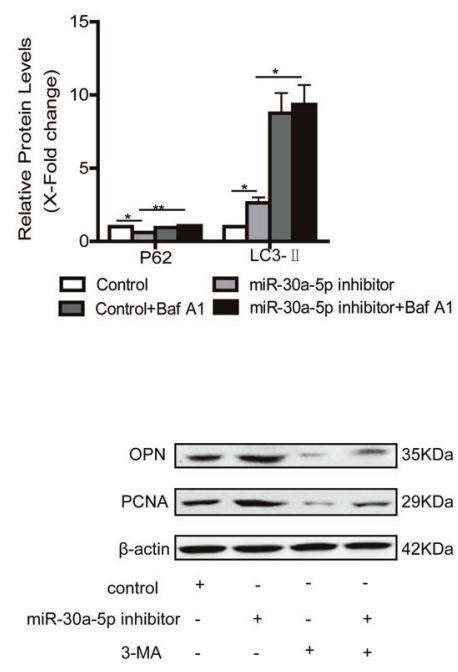

c
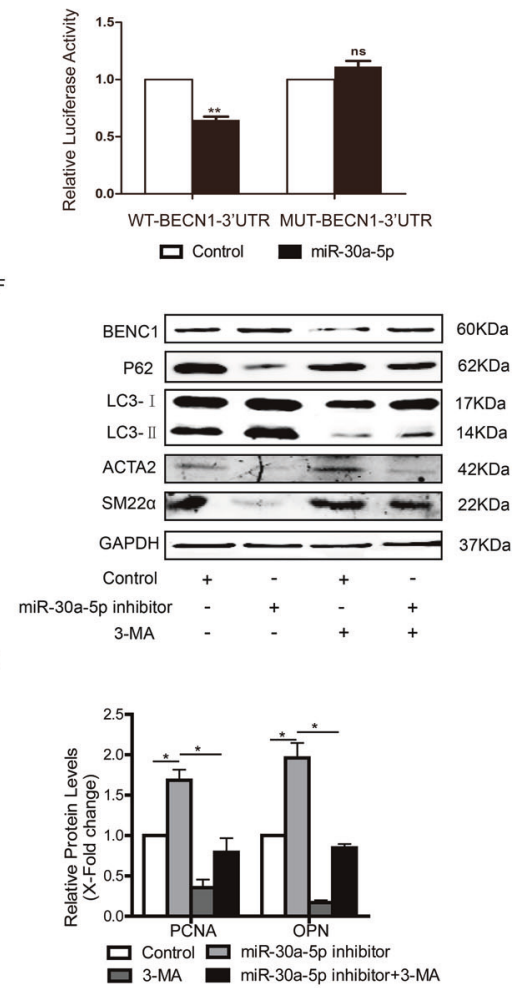

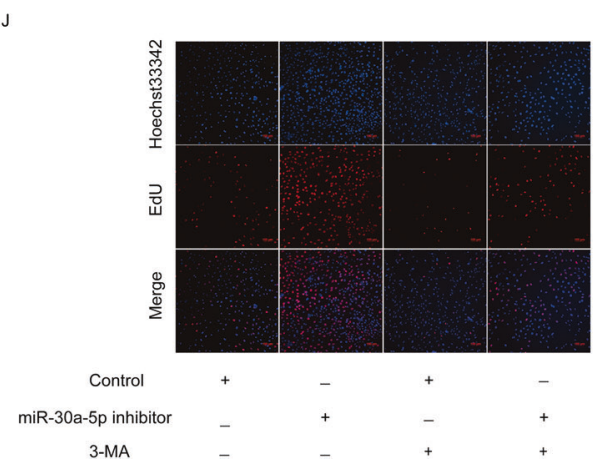

$\mathrm{K}$

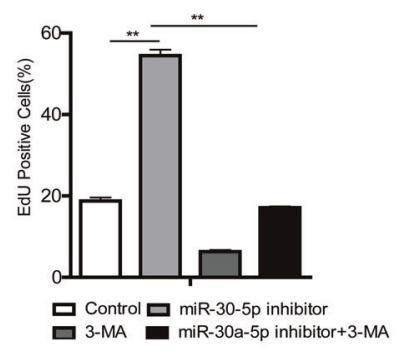

3-MA

Fig. 4 MiR-30a-5p decreased the expression of beclin1 by targeting the 3'UTR of beclin1. A HA-VSMCs were transfected with miR-30a-5p

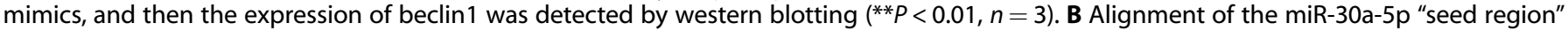

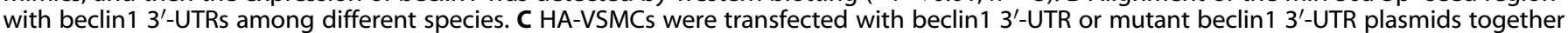
with miR-30a-5p mimic or control for $24 \mathrm{~h}$, followed by luciferase assays $\left({ }^{*} P<0.01, n=3\right)$. D and E HA-VSMCs were transfected with miR-30a$5 \mathrm{p}$ inhibitor or control for $24 \mathrm{~h}$ and then treated with bafilomycin A1 for $2 \mathrm{~h}$. Cells were harvested and accumulation of P62 and LC 3 was measured by western blotting ( $\left.{ }^{*} P<0.05,{ }^{*} P<0.01, n=3\right)$. F-I HA-VSMCs were transfected with miR-30a-5p inhibitor for $4 \mathrm{~h}$ and then treated

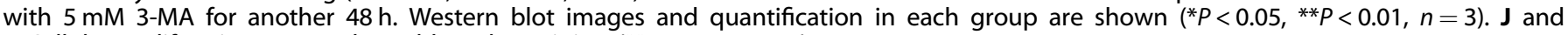
K Cellular proliferation was evaluated by EdU staining ( $\left.{ }^{*} P<0.01, n=3\right)$.

LC3-II and p62 expression, when bafilomycin A1 blocked lysosomal degradation (Fig. $5 \mathrm{H}$ and I). Inhibition of autophagy with 3-MA stabilized the expression of SM22a and ACTA2 in PDGF-BB-treated HA-VSMCs (Fig. 5J and K). These results indicated that PDGF-BB could induce HA-VSMC proliferation and trigger autophagy, while inhibition of autophagy could maintain the contractile phenotype.

\section{Restoring the expression of miR-30a-5p in PDGF-BB-treated cells upregulated the level of contraction proteins}

We next investigated the expression of miR-30a-5p in cells stimulated by PDGF-BB. As shown in Fig. 6A, miR-30a-5p was significantly decreased in PDGF-BB-stimulated cells. Because miR-30a-5p could inactivate autophagy, we hypothesized that inhibition of autophagy induced by miR-30a-5p could restore the levels of SM22a and ACTA2 in PDGF-BB-stimulated cells. As shown in Fig. 6B, miR-30a-5p reversed the effect of PDGF-BB in HA-VSMCs. MiR-30a-5p increased the accumulation of p62 and repressed the expression of beclin 1 and the ratio of $L C 3 I I / L C 3 I$, indicating the inhibition of autophagy. The loss of contractile proteins due to PDGF-BB treatment was resisted by miR-30a-5p. These results showed that miR-30a-5p could maintain the contractile phenotype of HA-VSMCs by inhibiting excessive autophagy.

Restoration of miR-30a-5p in the injured site abolished neointimal formation caused by wire injury of carotid arteries To further confirm the role of miR-30a-5p in VSMC phenotype switching, we performed a wire injury procedure on mouse carotid arteries, followed by local perivascular delivery of miR-30a- 
A
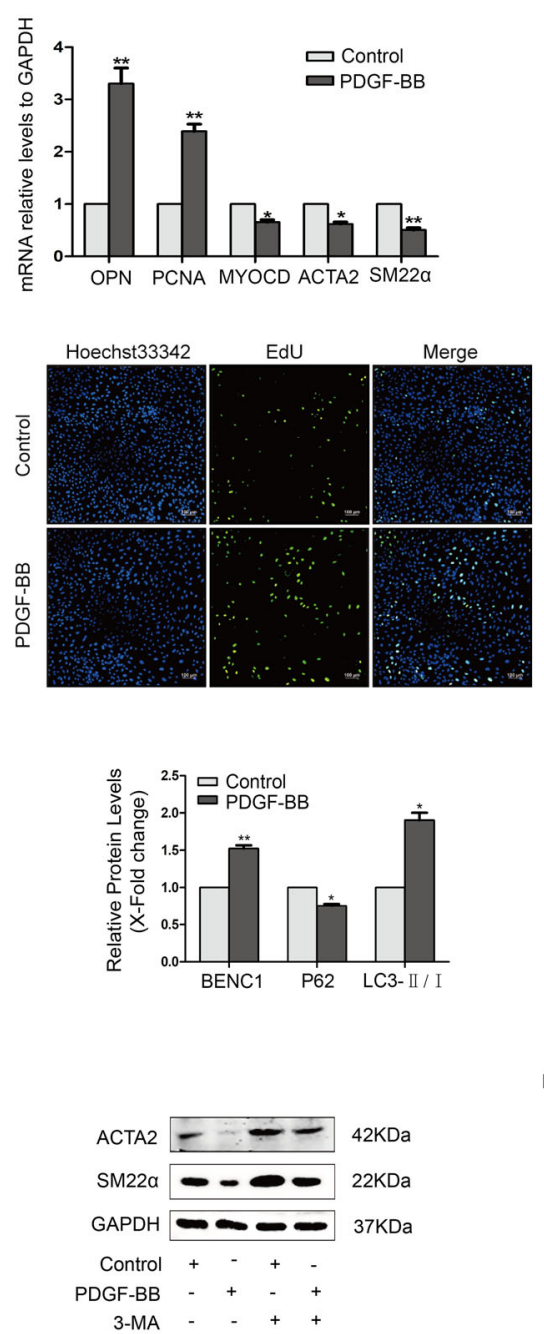

B

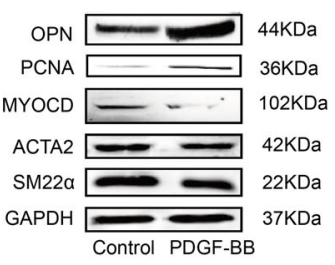

E

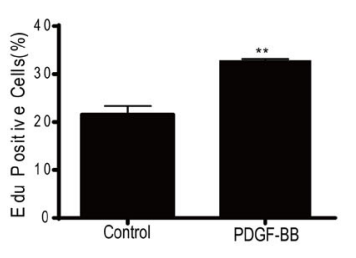

H

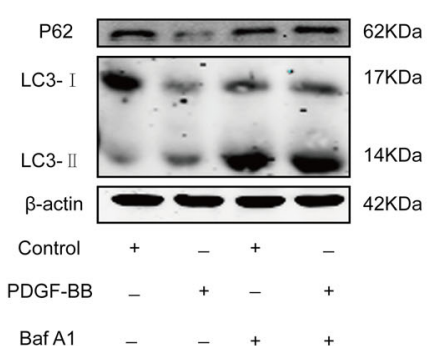

c
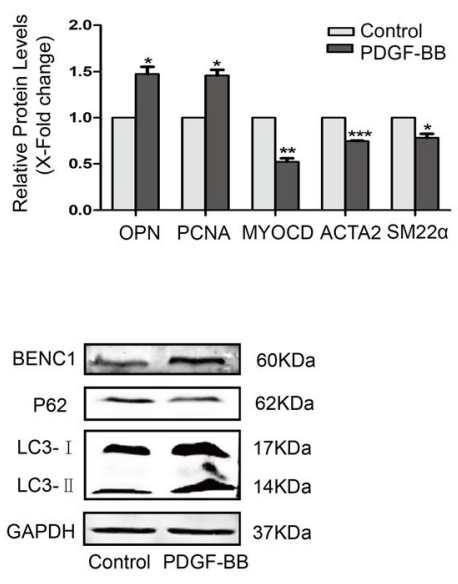

I

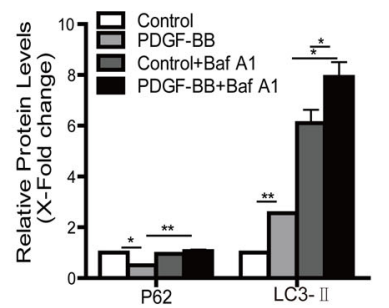

K

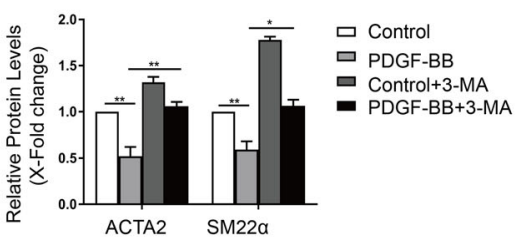

Fig. 5 Inhibition of autophagy maintained the contractile phenotype in PDGF-BB-stimulated HA-VSMCs. A HA-VSMCs were starved overnight prior to stimulation using $40 \mathrm{ng} / \mathrm{mL}$ PDGF-BB for $24 \mathrm{~h}$, and then the mRNA levels of OPN, PCNA, MYOCD, SM22 $\alpha$ and ACTA2 were detected by qRT-PCR $\left({ }^{*} P<0.05,{ }^{* * P}<0.01, n=3\right)$. B and $\mathbf{C}$ The protein levels of these proliferative markers and contractile genes were detected in HA-VSMCs treated with $40 \mathrm{ng} / \mathrm{mL}$ PDGF-BB for $48 \mathrm{~h}$ by western blotting $\left({ }^{*} P<0.05,{ }^{* *} P<0.01,{ }^{* * *} P<0.001, n=3\right)$. D and $\mathbf{E}$ Cellular proliferation was evaluated in PDGF-BB-stimulated HA-VSMCs by EdU staining $(* * P<0.01, n=3)$. F and $\mathbf{G}$ The expression of beclin 1 , P62 and LC3 was detected in PDGF-BB-stimulated HA-VSMCs by western blotting $\left({ }^{*} P<0.05,{ }^{* *} P<0.01, n=3\right)$. H and I HA-VSMCs were incubated with PDGF-BB for $24 \mathrm{~h}$ and then treated with bafilomycin A1 for $2 \mathrm{~h}$. Cells were harvested and accumulation of P62 and LC3 was measured by western blotting $\left({ }^{*} P<0.05,{ }^{*} P<0.01, n=3\right)$. J and $\mathbf{K}$ Serum-starved HA-VSMCs were pretreated with 5 mM 3-MA for half an hour and then incubated with $40 \mathrm{ng} / \mathrm{mL}$ PDGF-BB for another $48 \mathrm{~h}$. Western blot images and quantification in each group are shown $\left(* P<0.05,{ }^{*} P<0.01\right.$, $n=3)$.

$5 p$ agomiRs or scramble agomiRs. Three days or 21 days later, injured segments of femoral arteries were harvested and subjected to qRT-PCR and histochemistry staining. Three days after wire injury and local delivery of AgomiRs, the level of miR$30 a-5 p$ was detected in carotid arteries by qRT-PCR. Perivascular delivery of AgomiR-30a-5p significantly increased the levels of miR-30a-5p in the injured vessels (Fig. 7A). Then, we detected the expression of miR-30a-5p in the injured carotid arteries 21 days after wire injury. Importantly, the expression levels of miR-30a-5p were also significantly downregulated in the injured versus uninjured arteries (Fig. 7B). As shown in Fig. 7C, extensive neointimal growth was observed in sections of injured carotid arteries of mice treated with scramble AgomiRs compared with control mice. Treatment with agomiR-30a-5p effectively inhibited neointimal formation and the expression of LC3 and beclin-1 in the injured portion (Fig. 7C). AgomiR-30a-5p also increased the levels of MYOCD and ACTA2, and meanwhile decreased the levels of OPN. AgomiR-30a-5p decreased wire injury-induced neointimal hyperplasia, the intimato-media ratio and stenosis (Fig. 7D). In comparison with uninjured arteries, injured arteries treated with scramble AgomiRs displayed a significant increase in the level of the proliferation marker PCNA (Fig. 7E) and a decrease in the levels of the differentiation markers MYOCD and ACTA2 (Fig. 7F and G). Treatment with AgomiR-30a-5p restored the levels of MYOCD and ACTA2 and decreased smooth muscle cell proliferation, as confirmed by the decreased expression of PCNA (Fig. 7E-G). These results showed that restoration of miR-30a in the injured site inhibited the SMC phenotype switch and the neointimal formation caused by wire injury of carotid arteries.

\section{DISCUSSION}

By performing MYOCD loss-of-function studies, we demonstrated that MYOCD knockdown triggered autophagy in HA-VSMCs, as indicated by an increased LC3-II/LC3-I ratio, LC3 puncta formation and beclin 1 expression but decreased p62 accumulation. This is 

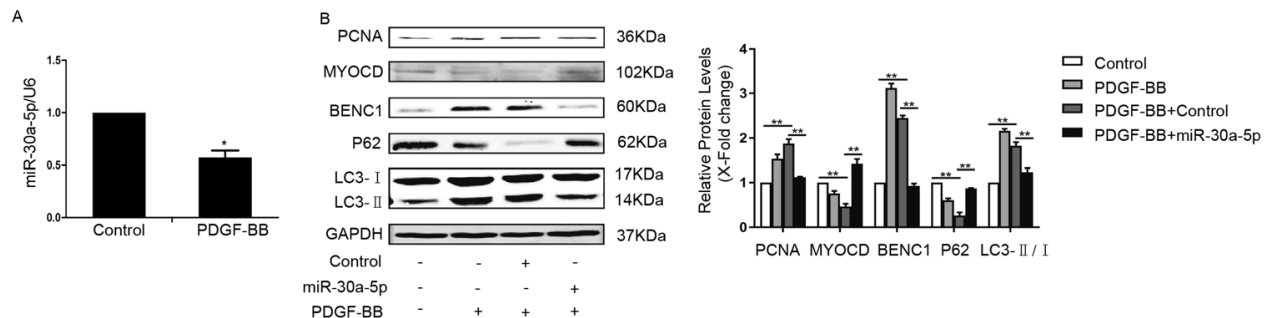

Fig. 6 Restoring the expression of miR-30a-5p in PDGF-BB-treated cells upregulated the level of contraction proteins. A Serum-starved HA-VSMCs were treated with $40 \mathrm{ng} / \mathrm{mL}$ PDGF-BB for $24 \mathrm{~h}$, and then the level of miR-30a-5p was detected by $\mathrm{qRT}-\mathrm{PCR}\left({ }^{*} P<0.05, n=3\right)$. B Serum-starved HA-VSMCs were transfected with miR-30a-5p mimics for $4 \mathrm{~h}$ and then stimulated with $40 \mathrm{ng} / \mathrm{mL}$ PDGF-BB for $48 \mathrm{~h}$. Western blot images and quantification in each group are shown $\left({ }^{*} P<0.05, * * P<0.01, n=3\right)$.

consistent with the recent literature showing that SMC-restricted MYOCD mutant mice exhibit activation of autophagy and deformation of the arterial structure [4]. MYOCD deletion can also trigger autophagy in primary cultures of mouse aortic SMCs in vitro, as confirmed by robust induction of beclin-1, autophagy related 5 (ATG5), (autophagy related 7) ATG7, and LC3 [4]. Simultaneously, MYOCD knockdown also led to downregulation of SMC contractile genes and the loss of the contractile SMC phenotype. Thus, we hypothesized that autophagy induced by MYOCD knockdown led to the degradation of SMC contractile proteins. For VSMCs, autophagy-lysosome, instead of ubiquitinproteasome, could be the major proteolytic pathway to remove contractile proteins [10].

PDGF-BB [10], TNF-a [9], nicotine [13], and shear stress [11] have been reported to promote VSMC phenotype switching and induce autophagy. These factor-induced phenotypic changes could depend on autophagy because inhibition of autophagy by treatment with 3-methyladenine (3-MA) reversed these effects and maintained the differentiation phenotype of VSMCs. Similarly, we found that MYOCD knockdown-mediated autophagy activation and VSMC phenotype switching were reversed by treatment with the autophagy inhibitor 3-MA. This result was coincident with several previous findings that activation of autophagy was observed in neointimal lesions of mouse common carotid arteries and that 3-MA prevented neointima formation [17]. Although our findings, together with other studies, have confirmed that deletion or knockdown of MYOCD could activate autophagy, the mechanism by which MYOCD regulates autophagy activities remains to be elucidated.

miR-30a-5p was found to suppress the expression of beclin 1 in hepatic stellate cells [18], cardiomyocytes [19-21], and a variety of tumor cells [14-16], leading to the inhibition of autophagy. A recent study confirmed that miR-30a inhibits autophagy and promotes VSMC senescence in rat aortic VSMCs by regulating the expression of beclin1 [22]. Consistent with a previous study, we demonstrated that beclin1 is a target of miR-30a-5p in HA-VSMCs. Using WT-3'UTR-BECN1 and mutant-3'UTR-BECN1 luciferase reporter vectors, we found that $m i R-30 a-5 p$ could repress the level of beclin 1 by targeting the $3^{\prime}$ UTR of beclin1. However, it is not known whether the expression of miR-30a-5p is regulated by MYOCD, a powerful transcriptional coactivator of SRF. By performing MYOCD loss-of function or gain-of function studies, we found that the expression of MYOCD was positively correlated with the level of miR-30a-5p in HA-VSMCs. We specifically showed that miR-30a-5p is transcriptionally regulated by MYOCD. MYOCD could activate the transcription of miR-30a-5p by binding to the CArG box of its promoter, as confirmed by luciferase reporter assays and ChIP. Thus, MYOCD could inactivate autophagy by activating miR-30a-5p and thus inhibiting beclin1.

To further clarify whether the effect of MYOCD in regulating autophagy depends on miR-30a-5p, miR-30a-5p mimics were transfected into MYOCD-knockdown cells. The data showed that miR-30a-5p mimics effectively restrained excessive autophagy induced by MYOCD knockdown and restored the expression of contractile proteins. It has been reported that miR-30a-5p could inhibit the activation of nuclear factor $\mathrm{KB}(\mathrm{NF}-\mathrm{KB})$ in many types of cells [23-25]. NF-KB activation has also been shown to inhibit myocardin expression and myocardin-mediated smooth muscle contractile marker genes [26-29]. Thus, miR-30a-5p could increase the levels of myocardin by inhibiting the activation of NF-KB. Furthermore, the miR-30a-5p inhibitor activated autophagy in HAVSMCs, and 3-MA treatment inhibited activated autophagy and resumed the expression of contractile proteins.

PDGF-BB is a potent mitogen for VSMCs, acting on the PDGFbeta receptor in VSMCs and promoting the migration and proliferation of VSMCs. MYOCD is inhibited by PDGF-BB signaling via transcription factors such as Kruppel-like factor $4[30,31]$ and ETS-like gene 1 (Elk-1) [31, 32] or miRNAs [33]. Several recent studies have suggested that autophagy is required for PDGF-BBinduced phenotypic conversion of SMCs [34-36]. PDGF-BB initiated autophagy in VSMCs through a $5^{\prime}$ adenosine monophosphate-activated protein kinase-independent and mammalian target of rapamycin-independent mechanism, leading to the rapid degradation of contractile proteins [10]. All three inhibitors of autophagy, i.e., spautin-1,3-MA and bafilomycin, prevented PDGF-BB-induced losses of contractile proteins and maintained the contractile phenotype [10]. In line with this study, our data showed that treatment with PDGF-BB enhanced the expression of the proliferative markers OPN and PCNA and reduced the levels of MYOCD, SM22a, and ACTA2 in HA-VSMCs. PDGF-BB induced cellular proliferation, as confirmed by EdU staining. This was accompanied by the activation of autophagy, characterized by an increase in beclin 1 and LC3II/LC3I and a decrease in p62. These effects of PDGF-BB were partially reversed by 3-MA.

Accumulating evidence show that autophagy activation in VSMCs is an essential regulator of the transition from a contractile to a synthetic phenotype, which contributes to neointima formation. High autophagy is observed not only in PDGFstimulated VSMCs in vitro but also in the VSMCs of injured arteries in vivo using a mouse partial left carotid artery (PLCA) ligation-injury model [37]. Treatment with 3-MA significantly alleviates PLCA ligation-induced neointima formation and decreased the cell proliferation marker PCNA in mice [37]. Furthermore, autophagy is overactivated in carotid arteries after balloon injury and that inhibition of autophagy activation contributes to preventing VSMC phenotype switching and hyperproliferation $[37,38]$. Jumonji domain-containing protein 3 (JMJD3) and mesoderm/mesenchyme homeobox gene I (Meox1) were found to be novel inducers of SMC phenotypic switching. Knockdown of JMJD3 or Meox1 attenuated injury-induced neointima formation and inhibited VSMC hyperproliferation in a rat carotid artery balloon injury model by suppressing autophagic activation.

However, basal autophagy levels are critically required for VSMC survival and vascular integrity during the development and 


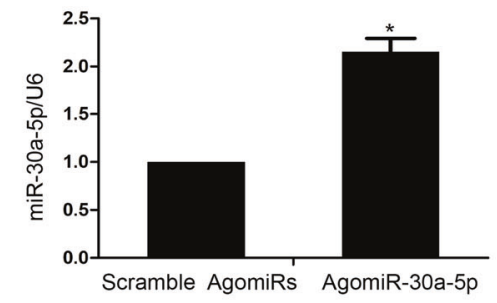

C

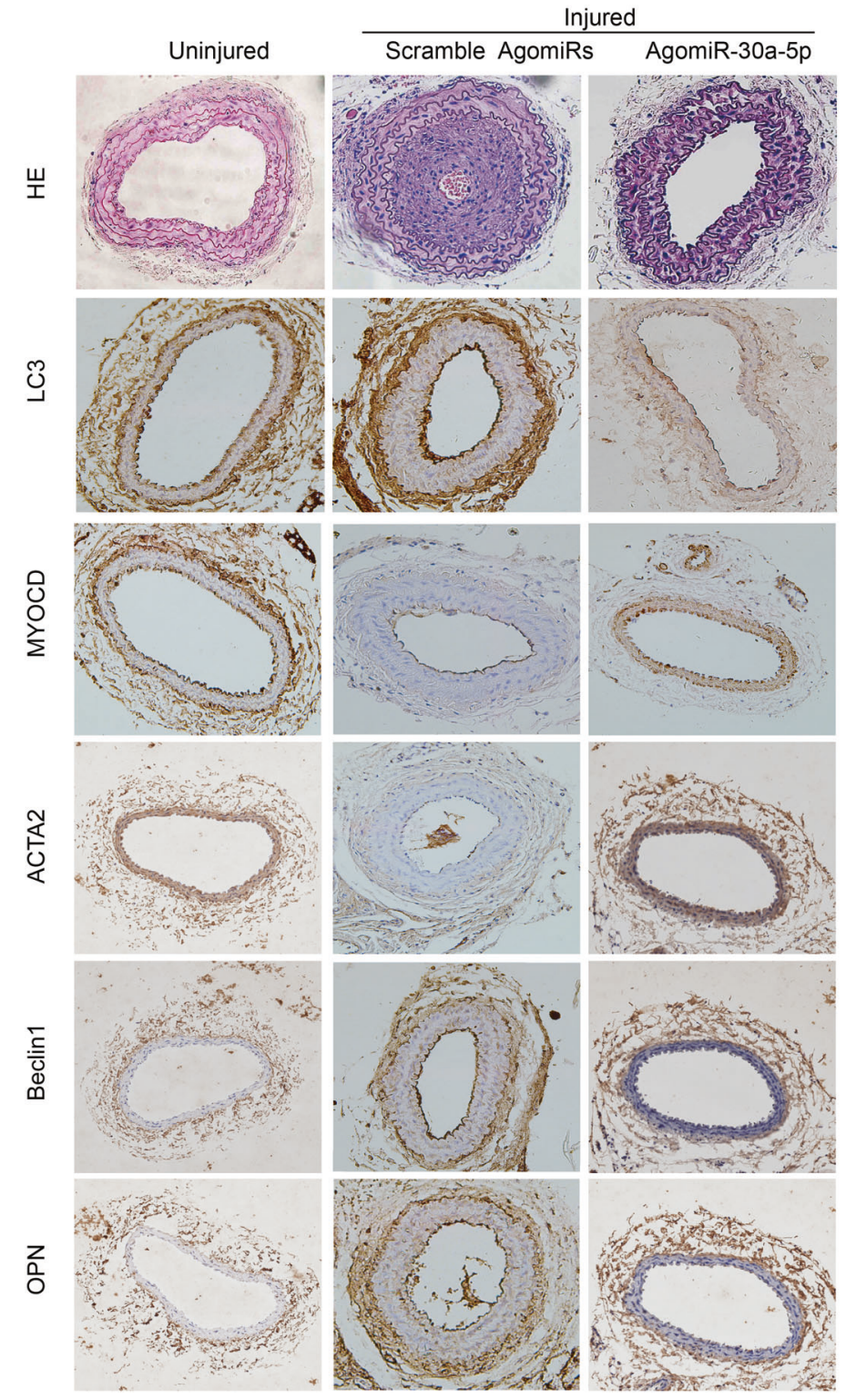

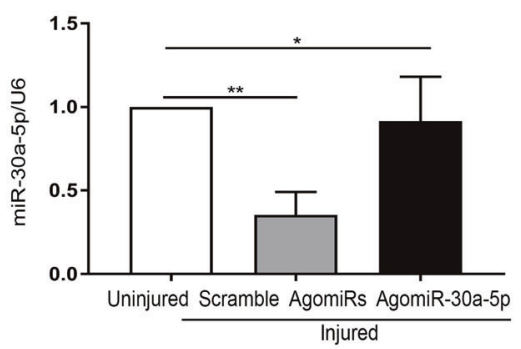

D

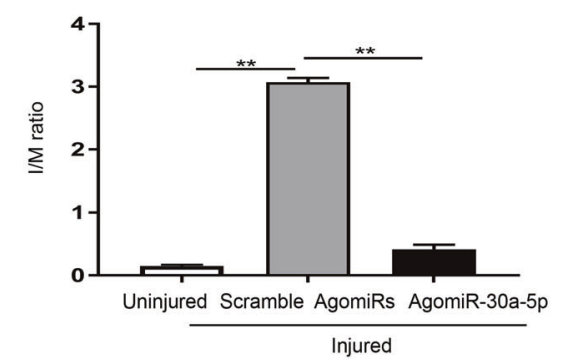

E

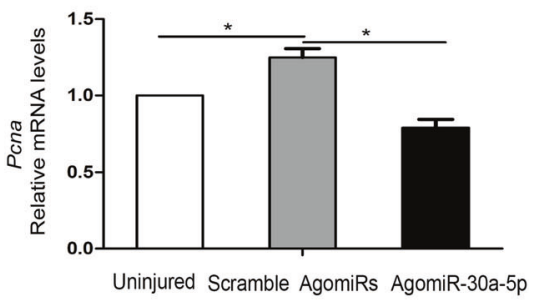

F
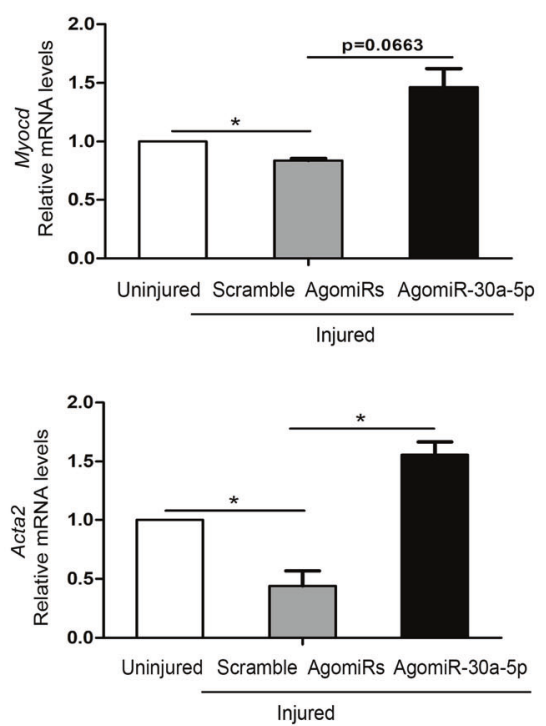

Fig. 7 Restoration of miR-30a-5p in the injured site abolished neointimal formation caused by wire injury of carotid arteries. Wire injury was performed in vivo on mouse carotid arteries, and then local perivascular delivery of miR-30a-5p agomiRs or scramble agomiRs was carried out. A Three days after injury, injured segments of femoral arteries were harvested, and qRT-PCR was performed to confirm the overexpression of miR-30a-5p $\left({ }^{*} P<0.05, \mathrm{n}=4-6\right)$. B At 21 days postinjury, injured segments of femoral arteries were harvested and subjected to qRT-PCR to detect the level of miR-30a-5p ( $\left.{ }^{*} P<0.05,{ }^{* *} P<0.01, n=4-6\right)$. C Representative images of HE staining and histochemistry staining. $\mathbf{D}$ Delivery of miR-30a-5p agomiRs led to a reduction in neointima size and a reduction in the intimato-media ratio $\left({ }^{* *} P<0.01, n=4-6\right)$. The mRNA levels of Pcna (E), Myocd (F) and Acta2 (G) were detected in injured mouse carotid arteries with miR-30a-5p agomiRs or scramble agomiRs by qRT-PCR $\left({ }^{*} P<0.05, n=4-6\right)$. 
progression of AAs. A recent study using $\mathrm{Tag} \ln C \mathrm{Cr}^{+} / \mathrm{Atg} 5^{\text {flox/flox }}$ mice suggested that loss of basal autophagy in VSMCs increased the susceptibility of VSMCs to death, enhanced ER stress activation, and promoted VSMC inflammation [7]. They also observed the induction of autophagy and endoplasmic reticulum stress in VSMCs of human dissecting AAs. Similarly, autophagic activity was increased in the aortic wall of thoracic aortic dissection patients [36]. Therefore, abnormal autophagy could regulate the functional properties of aortic SMCs, which might be associated with the potential pathogenesis of intimal hyperplasiarelated vascular diseases.

In the present study, we found that miR-30a-5p was significantly downregulated in PDGF-BB-treated HA-VSMCs in vitro and wire injury-induced mouse carotid arteries in vivo. Liu et al. observed that the expression of miR-30 family members, including miR-30ae, was significantly reduced in balloon-injured rat carotid arteries compared with uninjured rat carotid arteries [39]. Lentiviral delivery of miR-30c into injured carotid arteries prevented SMC proliferation and neointima formation by inhibiting the activation of CaMKIII2 [39]. Another study suggested that downregulation of miR-29b induced VSMC phenotypic modulation by directly activating ATG14-mediated autophagy, which was involved in the pathogenesis of intracranial aneurysm. Importantly, we found that restoring the expression of miR-30a-5p in PDGF-BB-treated HA-VSMCs could stabilize the expression of contractile proteins by inhibiting excessive autophagy. With a wire-injured mouse carotid artery model, we confirmed that local perivascular delivery of miR$30 a-5 p$ AgomiRs inhibited neointima formation by inhibiting the activation of autophagy.

In summary, we showed that MYOCD regulated the expression of miR-30a-5p, a potent inhibitor of autophagy, by downregulating beclin 1 expression. Our study provided the first evidence that MYOCD knockdown induced the degradation of VSMC contractile proteins by suppressing miR-30a-5p and activating autophagy. Restoring the expression of miR-30a-5p prevented PDGF-BBinduced VSMC phenotype modulation in vitro and inhibited VSMC proliferation in the arterial walls and neointimal hyperplasia in vivo following carotid injury. These studies provide a new potential therapeutic strategy to reduce the progression of intima hyperplasia-related vascular diseases.

\section{MATERIALS AND METHODS \\ Cell culture and treatment}

Human aortic VSMCs (HA-VSMCs) were obtained from the American Type Culture Collection (ATCC, Manassas, VA) and cultured in Dulbecco's modified high glucose medium (DMEM-HG) (Gibco, New York, USA) containing $10 \%$ fetal bovine serum (AusGeneX, Gold Coast, Australia) at $37^{\circ} \mathrm{C}$ in $5 \% \mathrm{CO}_{2}$ and $95 \%$ air.

HA-VSMCs were seeded onto 6 -well plates at $1 \times 10^{6}$ cells $/ \mathrm{mL}$. The cells were starved in serum-free media for another $24 \mathrm{~h}$ and then subsequently treated with $40 \mathrm{ng} / \mathrm{mL}$ PDGF-BB (R\&D, Minneapolis, USA) for $48 \mathrm{~h}$.

\section{Plasmid DNA and miRNA transfection}

A MYOCD-targeting shRNA plasmid (shMYOCD), a scrambled control shRNA plasmid (shControl), a pCDNA3.1-MYOCD expression plasmid (MYOCD) and an empty vector pcDNA3.1 (pcDNA3.1) have been described previously $[40,41]$. The GFP-LC3 plasmid was a gift from Congcong $\mathrm{He}$ (Northwestern University, Chicago, USA). Plasmid DNA transfection was carried out using X-tremeGENE HP DNA transfection reagent (Roche, Basel, Switzerland) according to the manufacturer's instructions. MiR-30a-5p mimics, inhibitors and miRNA negative controls were purchased from RiboBio (RiboBio, Guangzhou, China). MiRNA transfection was performed using riboFect ${ }^{\mathrm{TM}} \mathrm{CP}$ reagent according to the manufacturer's instructions.

\section{Western blotting}

Whole-cell proteins were extracted and subjected to standard western blot analysis as described previously [41]. Antibodies against MYOCD (SAB4200539, Sigma, Massachusetts, USA), ACTA2(ab7817, Abcam,
Massachusetts, USA), SM22a (ab14106, Abcam, Massachusetts, USA), PCNA (sc-56, Santa Cruz, California, United States), OPN (sc21742, Santa Cruz, California, United States), LC3 (NB100-2220, Novus Biologicals, Colorado, USA), P62 (610833,BD Transduction Laboratories, USA), Beclin1 (ab207612, Abcam, Massachusetts, USA) and glyceraldehyde-3-phosphate dehydrogenase (GAPDH) (ab8245, Abcam, Massachusetts, USA) were used to probe for the target proteins. Secondary antibodies, including IRDye $800 \mathrm{CW}$ antirabbit secondary antibody and IRDye 680 anti-mouse secondary antibody, were purchased from Li-COR (Lincoln, Michigan, USA). Signals were detected by an Odyssey ${ }^{\text {TM }}$ Infrared Imaging System (LI-COR, Michigan, USA), and the bands were quantified by Image-Pro Plus 5.1 software (MEDIA CYBERNETICS, Maryland, USA).

\section{Quantitative real-time PCR (qRT-PCR)}

Real-time PCR for mRNA and miRNA was performed as described in our previous studies in an Applied Biosystems real-time PCR system [41]. Briefly, total RNA was extracted using TRIzol reagent (Invitrogen, California, USA), and small RNA was extracted using a miRcute miRNA isolation kit (TIANGEN, Beijing, China) according to the manufacturer's instructions. CDNA was reverse transcribed from total RNA using M-MLV reverse transcriptase (Promega, Minnesota, USA) with random primers or specific primers for miR-30a-5p and U6. The sequences of reverse transcription primers were as follows: miR-30a5p: GTCGTATCCAGTGCAGGGTCCGAGGTATTCGCACTGGATACGACCTTCCAG U6:GTCGTATCCAGTGCAGGGTCCGAGGTATTCGCACTGGATACGACAAAAT ATG. Bestar ${ }^{\circledR}$ SybrGreen qPCR mastermix (DBI Bioscience, Ludwigshafen, Germany) was used for single-step qRT-PCRs. The results were standardized to control values of GAPDH or U6. The PCR primer sequences are listed in Table 1.

\section{Immunofluorescence assay}

For quantification of GFP-LC3 dots, HA-VSMCs were cotransfected with shMYOCD or shControl together with GFP-LC3 plasmid for $48 \mathrm{~h}$. The numbers of GFP-LC3 dots per cell were quantified from three independent experiments ( $\geq 100$ cells per experiment).

\section{Luciferase reporter assay}

For gene promoter activity assays, the promoter regions of miR-30a-5p were amplified by PCR and then cloned into the PGL3 luciferase reporter vector (WT-pmiR30a-5p-luc). The primers used were as follows: 5'-AGAGCTAGCGAG TAGTATAGGTCCCACTTGGAT-3' (sense) and 5'-CTTCTCGAGATACCTTCTTTAGC CTTCTGTTGGG-3' (antisense). Luciferase reporter constructs containing the CArG box-mutated hsa-miR-30a-5p promoter (MUT-pmiR30a-5p-luc) were generated using a QuikChange site-directed mutagenesis kit (Stratagene, La Jolla, CA). The primers used were as follows: $5^{\prime}$-GACTCAAAGATTAACTTITTTA AAAGGCATGGGATAGAGTTACCCTTTGCTTT-3' (sense); and 5'-AAAGCAAAGGG TAACTCTATCCCATGCCTITTAAAAAAGTTAATCTTTGAGTC-3' (antisense). The vector $\mathrm{pGL} 3$ was used as a control. VSMCs were cotransfected with gene promoter plasmids (WT-pmiR30a-5p-luc or MUT-pmiR30a-5p-luc) and the expression plasmids as indicated in the figure legends using X-tremeGENE HP DNA transfection reagent (Roche, Basel, Switzerland). Luciferase assays were performed using a luciferase assay kit (Promega, Minnesota, USA) according to the manufacturer's protocol. For beclin1 3'UTR reporter activity assays, luciferase-UTR reporter constructs (WT-BECN1-3'UTR) were generated by inserting the beclin $13^{\prime}$-UTR. The primers used for PCR were as follows: $5^{\prime}-$ GTTIAAACGAGCTCGCTAGCCTIITITCCTTAGGGGGAGGTTG-3' (sense) and 5'CTCTAGACTCGAGGCTAGCGGCAGTITTCAGACTGCAGCAAAT-3' (antisense). The beclin1 3'UTR-MUT luciferase reporter plasmid (MUT-BECN1-3'UTR) was constructed using the following primers: $5^{\prime}$-ATTCGGGTAATATTAAACAGTACA TAGGGCAAATACCAAAAAAGAAAAAATC-3' (sense) and 5'-GATTITTCTITITG GTATTTGCCCTATGTACTGTTTAATATTACCCGAAT- $3^{\prime}$ (antisense). HA-VSMCs were cotransfected with reporter plasmids (WT-BECN1-3'UTR or MUTBECN1-3'UTR) and control or miR-30a-5p mimics using X-tremeGENE HP DNA transfection reagent. After $24 \mathrm{~h}$, luciferase activity was measured with a Synergy ${ }^{\mathrm{Tm}} 4$ (Bioteck, Vermont, USA). Transfection efficiencies were normalized by the total protein concentrations of each sample.

\section{Chromatin immunoprecipitation (ChIP) assays}

ChIP analysis was performed as described previously [41]. The crosslinking reaction was terminated by glycine in MYOCD-transfected cells treated with $1 \%$ formaldehyde. The DNA fragments were broken into $200-1000 \mathrm{bp}$ fragments by ultrasonic disruption. Protein-DNA complexes were immunoprecipitated using primary antibodies against myc (MYOCD-myc) (Proteintech, Chicago, USA). Samples were immunoprecipitated with nonimmune rabbit IgG (Beyotime Biotechnology, Shanghai, China) as a 
Table 1. The PCR primer sequences.

\begin{tabular}{|ll|}
\hline Gene name & Sequence $\left(\mathbf{5}^{\prime} \rightarrow \mathbf{3}^{\prime}\right)$ \\
\hline U6 & F-ATTCAACGGCACAGTCAAGG \\
& R-GCAGAAGGGGCGGAGATGA \\
\hline Myocardin & F-CTCGCTTCGGCAGCACA \\
\hline PCNA & R-AACGCTTCACGAATTGCGT \\
\hline ACTA2 & F-AGTAAGAACCGCCACAAA \\
\hline OPN & R-GAGCATAGGCAGAGTCCA \\
\hline miR-30a-5p & F-GGCTCCATCCTCAAGAAGGTTT \\
\hline miR-30a-5p reverse transcription primer & R-CGTTATCTTCGGCCCTAGTGTA \\
\hline & F-AGCCAAGCACTGTCAGGAATC \\
\hline R-GAGCCCAGAGCCATTGTCAC \\
\hline F-AGTACCCTGATGCTACAGACGAG \\
\hline R-CGTTTCATAACTGTCCTTCCCAC \\
\hline
\end{tabular}

negative control. After washing and reversing the cross-links, the enriched DNA was purified and then quantified by real-time PCR. Primer sequences for amplification of the miR-30a-5p promoter containing one CArG box were as follows: $5^{\prime}$-ATAAAGAAAAAGGCCACA-3' (sense) and $5^{\prime}$ - AAAATC CACACAAAAAGC-3' (antisense).

\section{EdU staining}

Cell proliferation was determined by the incorporation of 5-ethynyl-20deoxyuridine (EdU) with a Cell-Light ${ }^{\mathrm{TM}}$ EdU DNA Cell Proliferation Kit (RiboBio, Guangzhou, China). Briefly, after being treated with $40 \mathrm{ng} / \mathrm{mL}$ PDGF-BB for $48 \mathrm{~h}$, the cells were incubated in $100 \mu \mathrm{L}$ of $50 \mu \mathrm{M}$ EdU for $2 \mathrm{~h}$, fixed with $4 \%$ paraformaldehyde, and stained with Hoechst 33342 . The cells were observed under a laser confocal microscope (OLYMPUS, Tokyo, Japan), and the rate of EdU-positive cells/total cells was counted.

\section{A mouse model of wire-injured carotid artery and perivascular delivery of miR-30a-5p agomiR}

Male C57BL/6J (8 weeks old) mice were purchased from the PLA Military Academy of Medical Sciences Laboratory Animal Center (Beijing, China) and were randomly divided into three groups. All animal procedures were performed in accordance with the National Institutes of Health Guide for the Care and Use of Laboratory Animals (the 8th Edition, revised in 2011) and approved by the Animal Ethics Committee of Tianjin University of Science and Technology. Wire-induced carotid artery injury was performed as described previously [3]. Briefly, mice were anesthetized by pentobarbital $(70 \mathrm{mg} / \mathrm{kg})$ via intraperitoneal injection. The left carotid artery of the anesthetized mouse was exposed and looped with silk sutures to temporarily stop the blood flow. A small incision was made, and a metal guide wire ( $0.381 \mathrm{~mm}$ in diameter) (Cook Inc., Bloomington, USA) was inserted into the artery and moved in and out 5 times with rotation to denude the endothelium. After vascular injury, local delivery of AgomiRs was performed according to previous reports [37]. Briefly, $100 \mu \mathrm{L}$ of $30 \%$ pluronic F-127 gel (Beyotime, Guangzhou, China) containing $2.5 \mathrm{nmol} \mathrm{miR-}$ $30 a-5 p$ or scramble AgomiRs was applied perivascularly to the injured carotid arteries for local delivery of AgomiRs. Twenty-one days after the injury, the mice were euthanized, and their carotid arteries were isolated and fixed with $4 \%$ paraformaldehyde or frozen directly in liquid nitrogen. The tissues were stained with hematoxylin and eosin for analysis of neointimal growth or stained with anti-LC3 antibody for evaluation of autophagy. The intima/media ratio of randomly selected sections of the carotid arteries was analyzed. QRT-PCR was performed to detect the mRNA levels of PCNA, MYOCD, and ACTA2.

\section{Statistical analysis}

All of the data were presented as the means \pm S.E.M. The differences between two experimental groups were analyzed by using a two-tailed Student's $t$ test or one-way ANOVA. $P<0.05$ was considered statistically significant and expressed as ${ }^{*} P<0.05,{ }^{* *} P<0.01,{ }^{* *} P<0.001$.

\section{DATA AVAILABILITY}

The data used to support the findings of this study are available from the corresponding author upon request.

\section{REFERENCES}

1. Wang D, Chang S, Wang Z, Sutherland L, Richardson JA, Small E, et al. Activation of cardiac gene expression by myocardin, a transcriptional cofactor for serum response factor. Cell. 2001;105:851-62.

2. Wang J, Li A, Wang Z, Feng X, Olson EN, Schwartz RJ. Myocardin sumoylation transactivates cardiogenic genes in pluripotent 10T1/2 fibroblasts. Mol Cell Biol. 2007;27:622-32.

3. Xia D, Zhou Z, Yu H, Zheng L, Tang K. Myocardin: a novel player in atherosclerosis. Atherosclerosis. 2017;257:266-78.

4. Huang J, Wang T, Wright AC, Yang J, Zhou S, Li L, et al. Myocardin is required for maintenance of vascular and visceral smooth muscle homeostasis during postnatal development. Proc Natl Acad Sci USA.2015;112:4447-52.

5. Kockx MM, De Meyer GR, Buyssens N, Knaapen MW, Bult H, Herman AG. Cell composition, replication, and apoptosis in atherosclerotic plaques after 6 months of cholesterol withdrawal. Circ Res. 1998;83:378-87.

6. Wolinsky H, Goldfischer S, Schiller B, Kasak LE. Lysosomes in aortic smooth muscle cells. Effects of hypertension. Am J Pathol. 1973;73:727-34.

7. Clément M, Chappell J, Raffort J, Lareyre F, Vandestienne M, Taylor AL, et al. Vascular smooth muscle cell plasticity and autophagy in dissecting aortic aneurysms. Arterioscler Thromb Vasc Biol. 2019;39:1149-59.

8. Scherz-Shouval R, Elazar Z. Regulation of autophagy by ROS: physiology and pathology. Trends Biochem Sci. 2011;36:30-38.

9. García-Miguel M, Riquelme JA, Norambuena-Soto I, Morales PE, Sanhueza-Olivares F, Nuñez-Soto C, et al. Autophagy mediates tumor necrosis factor-a-induced phenotype switching in vascular smooth muscle A7r5 cell line. PLoS One. 2018;13:e0197210.

10. Salabei JK, Cummins TD, Singh M, Jones SP, Bhatnagar A, Hill BG. PDGF-mediated autophagy regulates vascular smooth muscle cell phenotype and resistance to oxidative stress. Biochem J. 2013;451:375-88.

11. Sun L, Zhao M, Liu A, Lv M, Zhang J, Li Y, et al. Shear stress induces phenotypic modulation of vascular smooth muscle cells via AMPK/mTOR/ULK1-mediated autophagy. Cell Mol Neurobiol. 2018;38:541-8.

12. Li M, Wang N, Gong Q, Li Z, Liao H, Yang L, et al. $\mathrm{Ca}^{2+}$ signal-induced cardiomyocyte hypertrophy through activation of myocardin. Gene. 2015;557:43-51.

13. Wang Z, Liu B, Zhu J, Wang D, Wang Y. Nicotine-mediated autophagy of vascular smooth muscle cell accelerates atherosclerosis via nAChRs/ROS/NF-kB signaling pathway. Atherosclerosis. 2019;284:1-10.

14. Zhu H, Wu H, Liu X, Li B, Chen Y, Ren $X$, et al. Regulation of autophagy by a beclin 1-targeted microRNA, miR-30a, in cancer cells. Autophagy. 2009;5:816-23.

15. Fu T, Shi H, Zhou J, Peng F, Liu R, Shi M, et al. MicroRNA-30a suppresses autophagy-mediated anoikis resistance and metastasis in hepatocellular carcinoma. Cancer Lett. 2018;412:108-17.

16. Chen W, Li Z, Liu H, Jiang S, Wang G, Sun L, et al. MicroRNA-30a targets BECLIN-1 to inactivate autophagy and sensitizes gastrointestinal stromal tumor cells to imatini. Cell Death Dis. 2020;11:198. 
17. Chang J, Huang C, Hsueh Y, Wang W, Su C, Chang H, et al. Role of excessive autophagy induced by mechanical overload in vein graft neointima formation: prediction and prevention. Sci Rep. 2016;6:22147.

18. Chen J, Yu Y, Li S, Liu Y, Zhou S, Cao S, et al. MicroRNA-30a ameliorates hepatic fibrosis by inhibiting Beclin1-mediated autophagy. J Cell Mol Med. 2017:21:3679-92.

19. Guo R, Hu N, Kandadi MR, Ren J. Facilitated ethanol metabolism promotes cardiomyocyte contractile dysfunction through autophagy in murine hearts. Autophagy. 2012;8:593-608.

20. Wang J, Bie D, Sun F. Long noncoding RNA AK088388 regulates autophagy through miR-30a to affect cardiomyocyte injury. J Cell Biochem. 2019;120: 10155-63.

21. Wang $Y$, Hao $Y$, Zhang $H$, Xu L, Ding N, Wang R, et al. DNA hypomethylation of miR-30a mediated the protection of hypoxia postconditioning against aged cardiomyocytes hypoxia/reoxygenation injury through inhibiting autophagy. Circ J. 2020;84:616-25

22. Tan $P$, Wang $H$, Zhan J, Ma X, Cui $X$, Wang $Y$, et al. Rapamycin-induced miR-30a downregulation inhibits senescence of VSMCs by targeting Beclin1. Int J Mol Med. 2019;43:1311-20.

23. Zhao C, Wang M, An Y, Sun W, Li H, Li M. A positive feedback loop of miR-30a-5pWWP1-NF-KB in the regulation of glioma development. Int J Biochem Cell Biol. 2019;112:39-49.

24. Hu E, Ding L, Miao S, Liu F, Liu D, Dou H, et al. MiR-30a attenuates immunosuppressive functions of IL-1 $\beta$-elicited mesenchymal stem cells via targeting TAB3. FEBS Lett. 2015;589:3899-907.

25. Li B, Yao F, Ma Z, Chen B. MiR-30a-5p ameliorates LPS-induced inflammatory injury in human A549 cells and mice via targeting RUNX2. Innate Immun. 2021;27:41-49.

26. Liu Q, Liu Z, Zhou J, Cui L, Xu M. The long noncoding RNA NKILA protects against myocardial ischaemic injury by enhancing myocardin expression via suppressing the NF-KB signalling pathway. Exp Cell Res. 2020;387:111774.

27. Tang $H$, Zheng $L$, Callis TE, Stansfield WE, He J, Baldwin AS, et al. Myocardin inhibits cellular proliferation by inhibiting NF-kappaB (p65)-dependent cell cycle progression. Proc Natl Acad Sci USA. 2008;105:3362-7.

28. Liao H, Wang N, Zhao W, Zheng L, Zheng L, Xing J, et al. NF-KB (p65) negatively regulates myocardin-induced cardiomyocyte hypertrophy through multiple mechanisms. Cell Signal. 2014;26:2738-48.

29. Singh $P$, Zheng L. Dual regulation of myocardin expression by tumor necrosis factor-a in vascular smooth muscle cells. PLoS One. 2014;9:e112120.

30. Liu Y, Sinha S, McDonald OG, Shang Y, Hoofnagle MH, Owens GK. Kruppel-like factor 4 abrogates myocardin-induced activation of smooth muscle gene expression. J Biol Chem. 2005;280:9719-27.

31. Yoshida T, Kaestner KH, Owens GK. Conditional deletion of Krüppel-like factor 4 delays downregulation of smooth muscle cell differentiation markers but accelerates neointimal formation following vascular injury. Circ Res. 2008;102:1548-57.

32. Wang Z, Wang Z, Hockemeyer D, McAnally J, Nordheim A, Olson EN. Myocardin and ternary complex factors compete for SRF to control smooth muscle gene expression. Nature. 2004;428:185-9.

33. Davis BN, Hilyard AC, Nguyen PH, Lagna G, Hata A. Induction of microRNA-221 by platelet-derived growth factor signaling is critical for modulation of vascular smooth muscle phenotype. J Biol Chem. 2009;284:3728-38.

34. Wu C, Wang T, Lee SS, Kuo R, Wang C, Yen J, et al. Glucagon-Like peptide-1 receptor agonist attenuates autophagy to ameliorate pulmonary arterial hypertension through Drp1/NOX- and Atg-5/Atg-7/Beclin-1/LC3 $\beta$ pathways. Int J Mol Sci. 2019;20:3435.

35. Han JH, Park HS, Lee DH, Jo JH, Heo KS, Myung CS. Regulation of autophagy by controlling Erk1/2 and mTOR for platelet-derived growth factor-BB-mediated vascular smooth muscle cell phenotype shift. Life Sci. 2021;267:118978.

36. Wang Y, Zhao ZM, Zhang GX, Yang F, Yan Y, Liu SX, et al. Dynamic autophagic activity affected the development of thoracic aortic dissection by regulating functional properties of smooth muscle cells. Biochem Biophys Res Commun. 2016:479:358-64.

37. Luo X, Yang D, Wu W, Long F, Xiao C, Qin M, et al. Critical role of histone demethylase Jumonji domain-containing protein 3 in the regulation of neointima formation following vascular injury. Cardiovasc Res. 2018;114:1894-906.

38. Wu B, Zhang L, Zhu H, Zhang E, Zheng F, Yang Y, et al. Mesoderm/mesenchyme homeobox gene I promotes vascular smooth muscle cell phenotypic modulation and vascular remodeling. Int J Cardiol. 2018;251:82-89.
39. Liu F, Spinelli A, Sun Y, Jiang M, Singer DV, Ginnan R, et al. MicroRNA-30 inhibits neointimal hyperplasia by targeting $\mathrm{Ca}^{(2+)} /$ calmodulin-dependent protein kinase IIठ (CaMKIII). Sci Rep. 2016;20:26166.

40. Li M, Wang N, Gong Q, Li Z, Liao H, Yang L, et al. $\mathrm{Ca}^{2+}$ signal-induced cardiomyocyte hypertrophy through activation of myocardin. Gene. 2015;557:43-51.

41. Liao $\mathrm{H}$, Wang $\mathrm{N}$, Zhao W, Zheng L, Zheng L, Xing J, et al. STAT3 protein regulates vascular smooth muscle cell phenotypic switch by interaction with myocardin. J Biol Chem. 2015;290:19641-52.

\section{ACKNOWLEDGEMENTS}

We thank Assistant Professor Congcong He (Northwestern University, Chicago, USA) for providing the GFP-LC3 plasmid. This work was supported by the grants from National Natural Science Foundation of China (No. U20A20400), National Key R\&D Program of China (No. 2017YFD0400304), Innovative Research Team of Tianjin Municipal Education Commission (No. TD13-5015), and Tianjin Natural Science Foundation (No. 17JCZDJC33600)

\section{AUTHOR CONTRIBUTIONS}

DS, JD, and SX contributed equally to this work. NW and TZ contributed to the conception and design of the research and wrote the manuscript; DS, JD, SX, and LH performed the experiments and analysis of data. $\mathrm{HZ}$ and $\mathrm{XJ}$ provided analysis and interpretation of data and statistical analysis. $\mathrm{XR}, \mathrm{HH}, \mathrm{SZ}$, and $\mathrm{WM}$ provided technical support. All authors read and approved the final paper.

\section{COMPETING INTERESTS}

The authors declare no competing interests.

\section{ETHICS}

All animal procedures were performed in accordance with the National Institutes of Health Guide for the Care and Use of Laboratory Animals (the 8th Edition, revised in 2011) and approved by the Animal Ethics Committee of Tianjin University of Science and Technology.

\section{ADDITIONAL INFORMATION}

Supplementary information The online version contains supplementary material available at https://doi.org/10.1038/s41419-022-04588-0.

Correspondence and requests for materials should be addressed to Tongcun Zhang or Nan Wang.

Reprints and permission information is available at http://www.nature.com/ reprints

Publisher's note Springer Nature remains neutral with regard to jurisdictional claims in published maps and institutional affiliations.

Attribution 4.0 International License, which permits use, sharing, adaptation, distribution and reproduction in any medium or format, as long as you give appropriate credit to the original author(s) and the source, provide a link to the Creative Commons license, and indicate if changes were made. The images or other third party material in this article are included in the article's Creative Commons license, unless indicated otherwise in a credit line to the material. If material is not included in the article's Creative Commons license and your intended use is not permitted by statutory regulation or exceeds the permitted use, you will need to obtain permission directly from the copyright holder. To view a copy of this license, visit http://creativecommons. org/licenses/by/4.0/.

(c) The Author(s) 2022, corrected publication 2022 